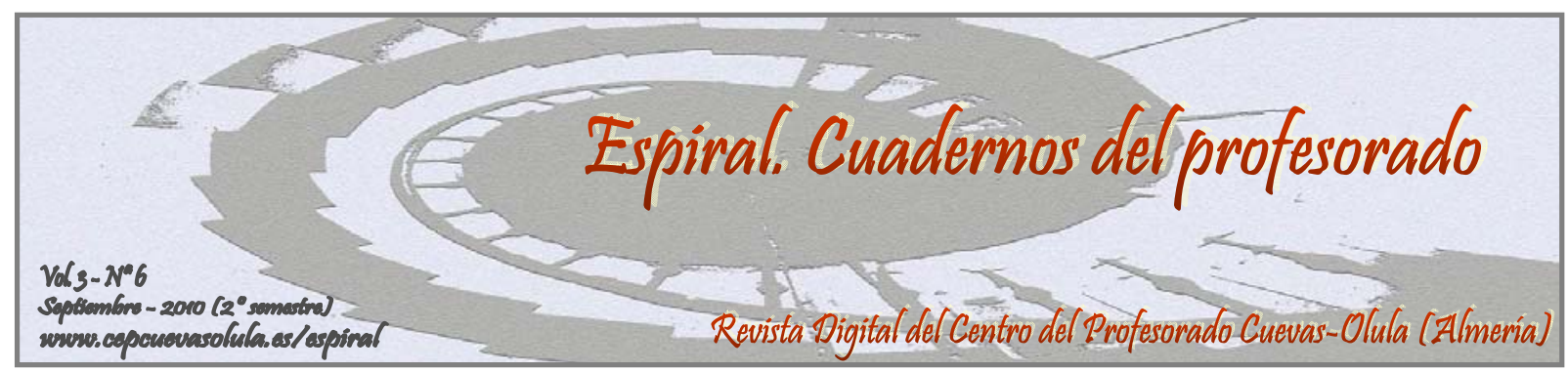

\title{
LA OPTIMIZACIÓN DEL PATIO DE RECREO PARA FAVORECER LA PRÁCTICA DE ACTIVIDADES FÍSICO-DEPORTIVAS MEDIANTE UN PROYECTO DE CONVIVENCIA
}

\section{THE OPTIMISATION OF THE PLAYGROUND IN ORDER TO FAVOUR ITS USE FOR PHYSICAL SPORTING ACTIVITIES BY MEANS OF A COMMUNITY PROJECT}

\author{
Marta García Rodríguez y José Miguel Serrano Aguilera
}

Centro de Educación Infantil y Primaria Mercedes Medina Díaz, Lanzarote, España

\begin{abstract}
RESUMEN: En este artículo pretendemos mostrar cómo a través de un proyecto de convivencia podemos motivar al alumnado hacia la práctica de actividades físicas, garantizando la participación activa de todos al optimizar los espacios que conforman el patio de recreo. Para ello partimos del proyecto de convivencia Optimización lúdica y regulación de la convivencia en el patio de recreo, que se está desarrollando en la actualidad en el Centro de Educación Infantil y Primaria Mercedes Medina Díaz de Lanzarote (Canarias). La realización de actividades físico-deportivas en un marco de convivencia multirracial garantiza la posibilidad de continuar educando al alumnado durante el recreo en valores para el desarrollo personal y social, tan necesarios en la sociedad actual. Además contamos con el carácter intrínseco de los juegos, es decir, jugar por jugar, lo que conlleva que el alumnado esté inmerso en su propio aprendizaje sin ser consciente de ello a través de situaciones lúdicas contextualizadas, facilitando así la elaboración y desarrollo de tareas competenciales, fundamentadas en la adquisición de las competencias básicas.
\end{abstract}

Palabras clave: Educación Física, valores, recreo, educación primaria, actividad físico-deportiva.

ABSTRACT: In this article we aim to show how, through a project of coexistence we can motivate the students towards the practice of physical activities, guaranteeing the active participation of all in order to optimise those spaces corresponding to the playground. In order to achieve this aim we star tout the project of coexistence Optimisation and Regulation of the Communal aspects in the playground, currently in development at the Infant and Primary Education Centre and the Mercedes Medina Diaz of Lanzarote (Canaries). The fulfilment of physical/sporting activities within the framework of a multiracial community guarantees the possibility of continuing the education of the student during playground in value towards their personal and social development, so necessary for real life. Furthermore we consider the intrinsic nature of the games that is, playing for playing's sake, which allows the student to be immersed in their own education, without being conscious of this due to the contextualised play situation, facilitating the work and development of tasks based on competences and, founded in the attainment of basic competencies.

Key words: Physical Education, value, playground, primary education, physical/sporting activity. 
García, M. \& Serrano, J.M. (2010). La optimización del patio de recreo para favorecer la práctica de actividades físico-deportiva mediante un proyecto de convivencia. Espiral. Cuadernos del Profesorado [en línea], 3(6), 32-42. Disponible en: http://www.cepcuevasolula.es/espiral.

Fecha de recepción: 04/12/2009

Fecha de aceptación: 18/04/2010
Enviar correspondencia a: martaprof76@hotmail.com

\section{1.- INTRODUCCIÓN.}

"En el proceso de desarrollo y avance de la sociedad, la educación se configura como un importante instrumento para impulsar la lucha contra las desigualdades, correspondiéndole al sistema establecer los mecanismos que contribuyan a prevenirlas y compensarlas, cualquiera que sea el motivo que las origine, y promover la transformación social a través de su compromiso solidario con las situaciones de desventaja en las que se encuentran colectivos y grupos que reciben los beneficios del sistema.” Ley 9/1999, de 18 de noviembre de Solidaridad en la Educación (BOJA no 140, 1999, 15429).

Vivimos en una sociedad pluralista en la que se mezclan las culturas, los estatus sociales y económicos, dejando al descubierto las desigualdades que existen entre unos y otros colectivos. Desde la escuela somos muchos los docentes que luchamos por paliarlas, ilusionados con la idea de que llegará el día en que la situación de inmigrante sólo suponga un enriquecimiento cultural para la población; no se trata sólo de aprender a respetar a otras culturas, sino de aprender a convivir con ellas. Para ello se hace preciso educar al alumnado en valores.

El término de educación en valores se implanta de forma específica con la Ley Orgánica 2/2006, de 3 de mayo, de Educación. En su artículo 1: Principios y fines de la educación, se establece como principio: "La transmisión y puesta en práctica de valores que favorezcan la libertad personal, la responsabilidad, la ciudadanía democrática, la solidaridad, la tolerancia, la igualdad, el respeto y la justicia, así como que ayuden a superar cualquier tipo de discriminación” (BOE n 106, 2006, 17164).

Así mismo, en su artículo 19 se recoge que: "Sin prejuicio de su tratamiento específico en algunas de las áreas de la etapa, la comprensión lectora, la expresión oral y escrita, la comunicación audiovisual, las tecnologías de la información y la comunicación y la educación en valores se trabajarán en todas las áreas". No obstante dicha ley no concreta cuáles son los valores que debemos trabajar con nuestro alumnado. Escamilla, Lagares y Fraile (2006), a través de su libro La LOE: perspectiva pedagógica e histórica: Glosario de términos esenciales, nos ofrecen algo de luz sobre esta cuestión al establecer como contenidos de valor para el desarrollo personal-social del alumnado el autoconocimiento y la autoestima, la educación moral y cívica y para la paz y la convivencia, la educación para la igualdad de oportunidades entre sexos, la educación para la salud, la educación ambiental, la educación sexual, la educación vial y la educación para el consumo.

De todo ello se desprende que nuestra labor educativa no debe ceñirse únicamente a la transmisión de contenidos conceptuales, sino también actitudinales, valiéndonos para ello de los procedimentales. Además al educar en valores al alumnado estamos contribuyendo con la adquisición de la competencia básica social y ciudadana, tan necesaria en la actualidad.

Basándonos en los argumentos ya expuestos, surge en nuestro centro la propuesta de desarrollar un proyecto que convivencia que mejore las relaciones entre el alumnado, evitando la formación de guetos y posibilitando la participación de todos ellos en la práctica de actividades físico-deportivas.

Para ello la idea principal gira en torno a la optimización del patio durante el recreo, ya que es el momento donde mayor cantidad de conflictos se producen, y en permitir al alumnado hacer uso del material deportivo del centro, puesto las desigualdades económicas entre ellos propicia que únicamente los más favorecidos puedan traer de sus hogares material deportivo para este tiempo lectivo, no permitiendo muchas veces la participación en el juego a otros compañeros, generando así más conflictos. 
Los maestros especialistas en Educación Física somos conscientes de que podemos contribuir en gran medida en el desarrollo de este proyecto, ya que a diario hacemos uso de esos espacios y utilizamos el juego como estrategia metodológica, y no únicamente como contenido; según el Decreto 126/2007, de 24 de mayo, por el que se establece la ordenación y el currículo de la Educación Primaria en la Comunidad Autónoma de Canarias, "El juego motor, del que nuestra Comunidad dispone de un legado que conviene reconocer, vivenciar, valorar y conservar es, asimismo, un recurso metodológico imprescindible en la Educación Primaria. De esta manera, se configura como un medio óptimo para que se adquieran gran parte de los contenidos del área" (BOC n 112, 2007, 12722).

Por otro lado, es importante recordar que un buen profesor de Educación Física es aquel que se implica en las actividades del centro.

En palabras de Sebastiani i Obrador y Delgado (2009); “Un buen profesor de Educación Física debe estar integrado e implicado totalmente en el centro donde trabaja. Éste es un aspecto muy valorado por los dirigentes de las escuelas y por los gestores educativos, pues lo consideran un buen profesional en la medida en que se integra, es coherente y forma parte activa y constructiva dentro de un proyecto educativo que, más allá del reducto de su asignatura, recae en la totalidad de la escuela” (p. 194).

No obstante, para el éxito de este proyecto es necesaria la contribución del resto del profesorado y la implicación activa por parte del alumnado, los cuales a día de hoy continúan participando con agrado.

A través de este artículo pretendemos mostrar cómo a través de un proyecto de convivencia podemos motivar al alumnado hacia la práctica de actividades físico-deportivas, garantizando la participación activa de todos al optimizar los espacios que conforman el patio de recreo.

\section{2.- PRESENTACIÓN DEL PROYECTO.}

\section{Contextualización.}

Nuestro proyecto se está desarrollando en la actualidad en el Centro de Educación Infantil y Primaria Mercedes Medina Díaz, situado en el extrarradio de Arrecife (Lanzarote). Carece de gimnasio y salón de actos, pero dispone de dos pistas polideportivas, abundante material de
Educación Física y una amplia zona de recreo correctamente asfaltada.

Su alumnado procede en su mayoría de los barrios de Tinasoria y Altavista. A él acuden insulares, peninsulares, subsaharianos y, sobre todo, sudamericanos, cuyos padres trabajan en diferentes sectores, como la construcción y la hostelería, factor que influye en la existencia de un nivel socioeconómico bajo que se agrava además por las situaciones irregulares de algunos de ellos, la desestructuración familiar y la crisis económica por la que está pasando la isla.

\section{Descripción del proyecto.}

El proyecto "Optimización lúdica $y$ regulación de la convivencia en el patio de recreo" comienza en el año 2005 y se crea con la finalidad de optimizar el espacio del patio durante el recreo y mejorar las relaciones que en él se producen. Este proyecto se divide en dos fases claramente diferenciadas pero vinculadas entre sí, compartiendo objetivos comunes que garantizan su coherencia en el paso del tiempo. Cada una de estas fases se subdivide en una serie de pasos a fin de marcar unas pautas de trabajo que parten siempre de los resultados del paso anterior (figura 1).

La primera fase, que recibe el nombre de: Aquí jugamos todos/as comenzó en el curso escolar 2005-2006 y a día de hoy continúa llevándose a cabo al tratarse de un trabajo de carácter abierto, ya que cada año se tienen en cuenta los nuevos intereses del alumnado, por lo que es sometido a revisiones periódicas continuas en las que se van modificando las actividades físico-deportivas que se proponen, garantizando así la motivación de los alumnos/as. De acuerdo con Baena, Calvo y Martínez (2009, p. 47), "lo más importante para un buen aprendizaje es la motivación y el interés del niño".

La segunda fase: Con vivir no basta, aprendemos a convivir jugando, se crea con la intención de que sea el propio alumnado el que regule la convivencia y cuide de las relaciones interpersonales en el patio de recreo.

En palabras de Vila (2009, p.47), "no se trata de tolerar al otro/a sin más, sino de ser causa y efecto para la coexistencia en un espacio de relaciones que debe generarse desde la cooperación, el consenso y el reconocimiento del otro/a como fuente de derechos". 


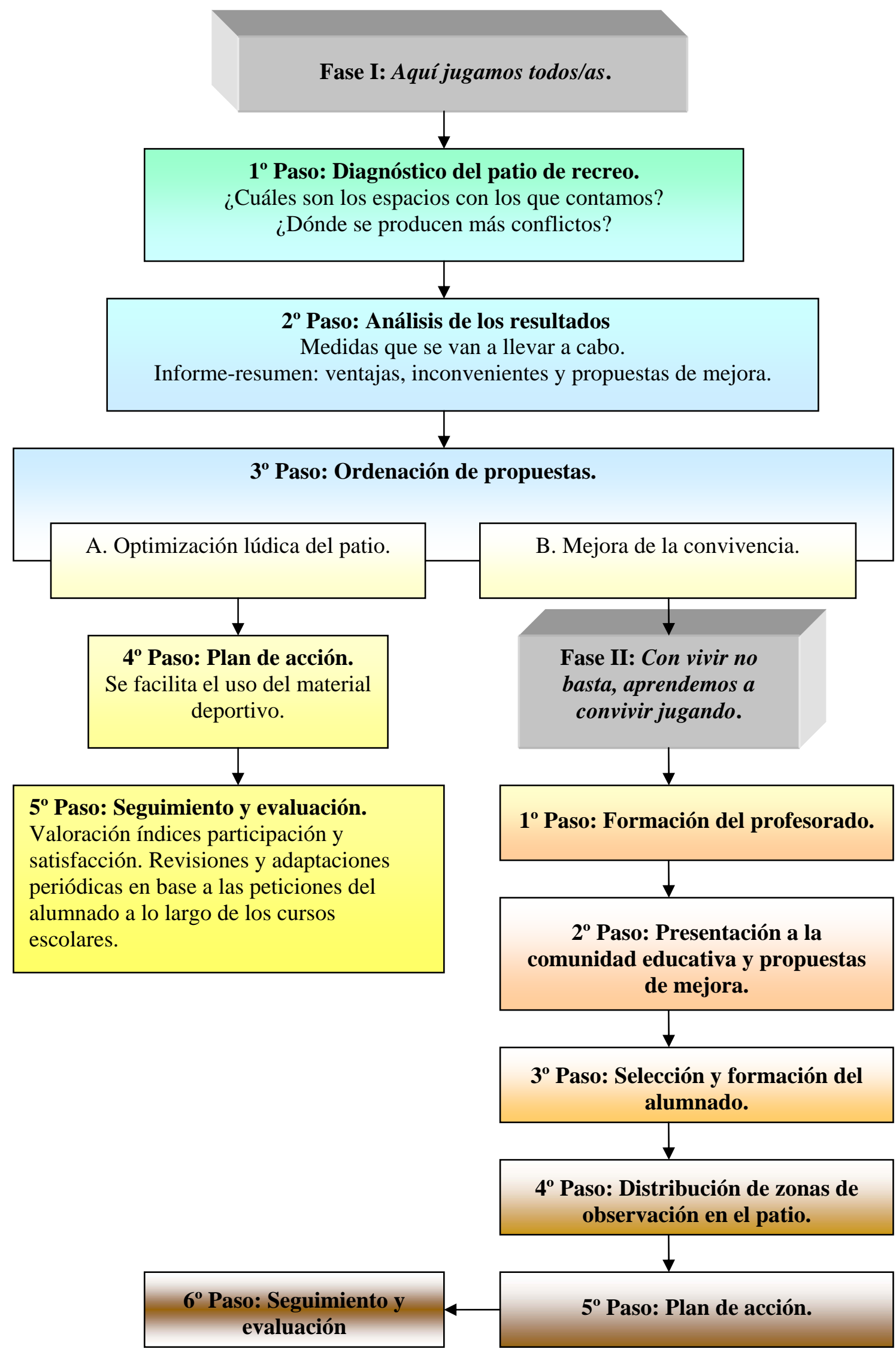

Figura 1.- Fases del proyecto 
El profesorado es, por norma general, el encargado de solucionar los conflictos que se generan de forma habitual en el patio de recreo, incluyendo en el término conflictos desde los más insignificantes hasta los más graves. Esto siempre ha sido así porque habitualmente nos olvidamos de algo muy importante, del mayor recurso del que disponemos, el recurso humano: el propio alumnado. Creemos firmemente que la regulación de la convivencia es posible, que la ayuda entre iguales funciona, sólo tenemos que encaminar al alumnado y guiarlo un poquito. Para ello, de los diferentes tipos de ayuda entre iguales llevados a buen término en escuelas europeas, vamos a aplicar con nuestro alumnado en la fase II del proyecto el denominado "Mediation and conflict resolution" (Mediación y resolución de conflictos). Tal como nos indican Cowie y Fernández (2006, p. 296), "se trata de un proceso estructurado en el cual los alumnos voluntarios son entrenados para ayudar a resolver conflictos entre sus compañeros. Los mediadores ayudan a sus compañeros a encontrar una solución satisfactoria para ambas partes".

\section{Objetivos del proyecto.}

\section{- Fase I:}

- Iniciar un proyecto para mejorar los recursos y el ambiente de convivencia en el patio de recreo, considerando prioritarias las ideas propuestas por el alumnado.

- Dinamizar el espacio del patio dedicado al fútbol, vivenciándolo de forma más participativa y responsable, valorando el espacio para este juego y cuantas personas lo usan.

- Valorar las posibilidades educativas que nos brinda el recreo.

- Propiciar en los alumnos y alumnas actitudes comprometidas hacia el cuidado y mejora de un espacio compartido.

- Mejorar las relaciones entre el alumnado.

- Fase II:

- Fomentar la colaboración, el conocimiento y búsqueda de soluciones en problemas interpersonales en el ámbito escolar.

- Disminuir la conflictividad y, con ello, la aplicación de medidas sancionadoras.

- Favorecer la participación directa del alumnado en la resolución de conflictos en la escuela.
- Crear canales de comunicación y de conocimiento mutuo entre educadores $\mathrm{y}$ educadoras y alumnado, mejorando la autoestima de todos los participantes en el proyecto.

- Contribuir a la mejora de la convivencia en el centro educativo.

\section{3.- DESARROLLO DEL PROYECTO.}

- Fase I: Aquí jugamos todos/as.

Primer paso: Diagnóstico del patio de recreo.

- Se establece como prioridad realizar un estudio del espacio que es más utilizado por los alumnos durante el recreo a través de cuestionarios, encuestas y asambleas, prestándose especial atención a la pista de fútbol, ya que es donde pensamos que es más fácil optimizar el espacio y donde mayor número de conflictos se producen.

- Se crea una comisión de seguimiento compuesta por la Junta de Delegados/as, la Directora del centro y el Coordinador del Proyecto (siendo éste un maestro especialista en Educación Física).

\section{Segundo paso: Análisis de los resultados.}

- Se examinan los resultados obtenidos y recogidos a través de los diferentes instrumentos empleados, se realiza un vaciado y se exponen los datos obtenidos a fin de ser sometidos a debate.

- Cada grupo-clase analiza los datos obtenidos, donde se reflexiona, debate y se consensúa qué aspectos se consideran más importantes, así como las medidas que se llevarán a cabo y cuál será el nivel de implicación del alumnado.

- Se elabora un informe-resumen con las ventajas, inconvenientes y las propuestas de mejora para cada una de las zonas del patio sometidas a análisis, obteniendo los mayores resultados en lo que concierne a la pista de fútbol (figura 2).

\section{Tercer paso: Ordenación de propuestas.}

A partir de aquí el proyecto toma dos caminos diferentes:

A. La optimización lúdica del patio.

B. La mejora de las relaciones interpersonales que se producen en el recreo, que se espera que mejoren con la implantación del punto anterior, y que por tanto tendría un proyecto específi- 
co enmarcado dentro del Proyecto de Convivencia del Centro.

Nos centramos inicialmente en la parte A del proyecto, donde se tratará de optimizar de la manera más lúdica posible el patio de recreo, a través de la inclusión de materiales y zonas delimitadas para su uso.

Se acuerdan en la Junta de Delegados/as los compromisos que están dispuestos a adquirir para conseguir y mantener esas mejoras lúdicas que harán que el tiempo de recreo sea más educativo, más divertido y menos conflictivo.

\section{Cuarto paso: Plan de acción.}

- Se rediseña el espacio y se crean las diferentes zonas de juegos (se pinta en el suelo una pista de voleibol, un campo específico para el brilé, diferentes círculos a modo de bases para jugar al béisbol, varias zonas destinadas al tres en raya y

\begin{tabular}{|c|c|c|}
\hline Propuesta de mejora & Responsables & Recursos necesarios \\
\hline $\begin{array}{l}\text { Delimitar bien el espacio empleado para } \\
\text { jugar al fútbol. }\end{array}$ & Profesorado y alumnado & Los existentes. \\
\hline $\begin{array}{l}\text { Realizar unas clases teóricas donde se } \\
\text { enseñen las normas básicas para jugar a } \\
\text { este deporte. }\end{array}$ & $\begin{array}{c}\text { Profesorado de Educación } \\
\text { Física }\end{array}$ & $\begin{array}{l}\text { Organizar las sesiones de clase } \\
\text { para recibir puntualmente } \\
\text { información }\end{array}$ \\
\hline $\begin{array}{l}\text { Elegir los capitanes/as por consenso del } \\
\text { equipo, debiendo estar formadas para } \\
\text { llevar a cabo su labor de forma óptima. }\end{array}$ & $\begin{array}{l}\text { No habrá capitanes/as. } \\
\text { Serán los tutores. } \\
10 \text { equipos/10 tutores. }\end{array}$ & Llevar a cabo un acuerdo. \\
\hline $\begin{array}{c}\text { Establecer las pautas para formar los } \\
\text { equipos. }\end{array}$ & Equipos heterogéneos. & $\begin{array}{c}\text { Criterios de elaboración de los } \\
\text { equipos. }\end{array}$ \\
\hline $\begin{array}{l}\text { Establecer con claridad el tiempo de } \\
\text { inicio y de finalización del partido. }\end{array}$ & $\begin{array}{c}\text { El árbitro. Dos tiempos de } \\
10 \text { minutos y descanso } \\
\text { intermedio. }\end{array}$ & $\begin{array}{l}\text { Silbato, cronómetro, balón de } \\
\text { fútbol. }\end{array}$ \\
\hline $\begin{array}{c}\text { Posibilidad de formar equipos entre los } \\
\text { diferentes grupos dentro de un mismo } \\
\text { curso. }\end{array}$ & Los tutores/as. & $\begin{array}{l}\text { Criterios de formación de } \\
\text { equipos. }\end{array}$ \\
\hline Nombrar responsables del material. & $\begin{array}{l}\text { Docente de guardia en el } \\
\text { porche (con carácter } \\
\text { rotatorio). }\end{array}$ & $\begin{array}{l}\text { Según la actividad a } \\
\text { desarrollar. }\end{array}$ \\
\hline $\begin{array}{l}\text { El árbitro será el responsable de realizar } \\
\text { el resumen de incidencias ocurridas } \\
\text { durante el partido. }\end{array}$ & Árbitros. & Cuaderno de actas. \\
\hline $\begin{array}{l}\text { Penalizar sin jugar al equipo que no } \\
\text { cumpla las normas establecidas. }\end{array}$ & $\begin{array}{l}\text { Árbitro (con el visto bueno } \\
\text { del profesor de EF o de la } \\
\text { Directora). Faltas } \\
\text { reiteradas y graves. }\end{array}$ & Actas para su valoración. \\
\hline $\begin{array}{l}\text { Aprender a aceptar los resultados con } \\
\text { deportividad. }\end{array}$ & El alumnado participante. & $\begin{array}{l}\text { Cariñograma. } \\
\text { Felicitar cada día a tres. }\end{array}$ \\
\hline $\begin{array}{l}\text { Organizar campeonatos entre alumnos y } \\
\text { alumnos-profesores }\end{array}$ & El comité. & Tiempo disponible. \\
\hline $\begin{array}{c}\text { Creación del “rincón para la reflexión” } \\
\text { para aquellos que muestren actitudes } \\
\text { incorrectas }\end{array}$ & Los tutores/as. & Especificar el espacio. \\
\hline Poner las redes a las porterías. & $\begin{array}{l}\text { Equipo directivo - } \\
\text { Ayuntamiento. }\end{array}$ & Redes y solicitud. \\
\hline
\end{tabular}

Figura 2.- Propuestas de mejora para la pista de fútbol. 
un tablero de ajedrez).

- Se adquiere nuevo material deportivo y didáctico.

- Se realiza una selección de los responsables de la entrega y recogida de dicho material, así como del uso del mismo durante el recreo.

- Se crea una comisión específica integrada por representantes de todos los colectivos implicados para su seguimiento y evaluación.

- Se pone en práctica el proyecto.

\section{Quito paso: Seguimiento y evaluación.}

- Se elabora un informe con las mejoras producidas y se valoran los índices de participación y satisfacción por parte del alumnado (se observa una bajada notable de los conflictos entre el alumnado desde la inclusión del material deportivo en el recreo, una mayor participación y un au- mento de la satisfacción del alumnado).

- Se aprueba por la comisión específica formada la continuidad de esta parte del proyecto a lo largo de los venideros cursos escolares, llevándose para ello a cabo seguimientos y evaluaciones constantes del plan de optimización del recreo, modificándose zonas y pautas de conducta en base a los resultados que se van obteniendo y a las nuevas propuestas de los alumnos/as para realizar otras posibles actividades físicas lúdicas.

- Fase II: Con vivir no basta, aprendemos a convivir jugando.

Primer paso: Formación del profesorado.

- Aunque muchos de los docentes del centro ya han realizado algún curso de formación sobre convivencia escolar, se aprueba realizar otros cursos específicos sobre este tema ofrecidos por el Centro

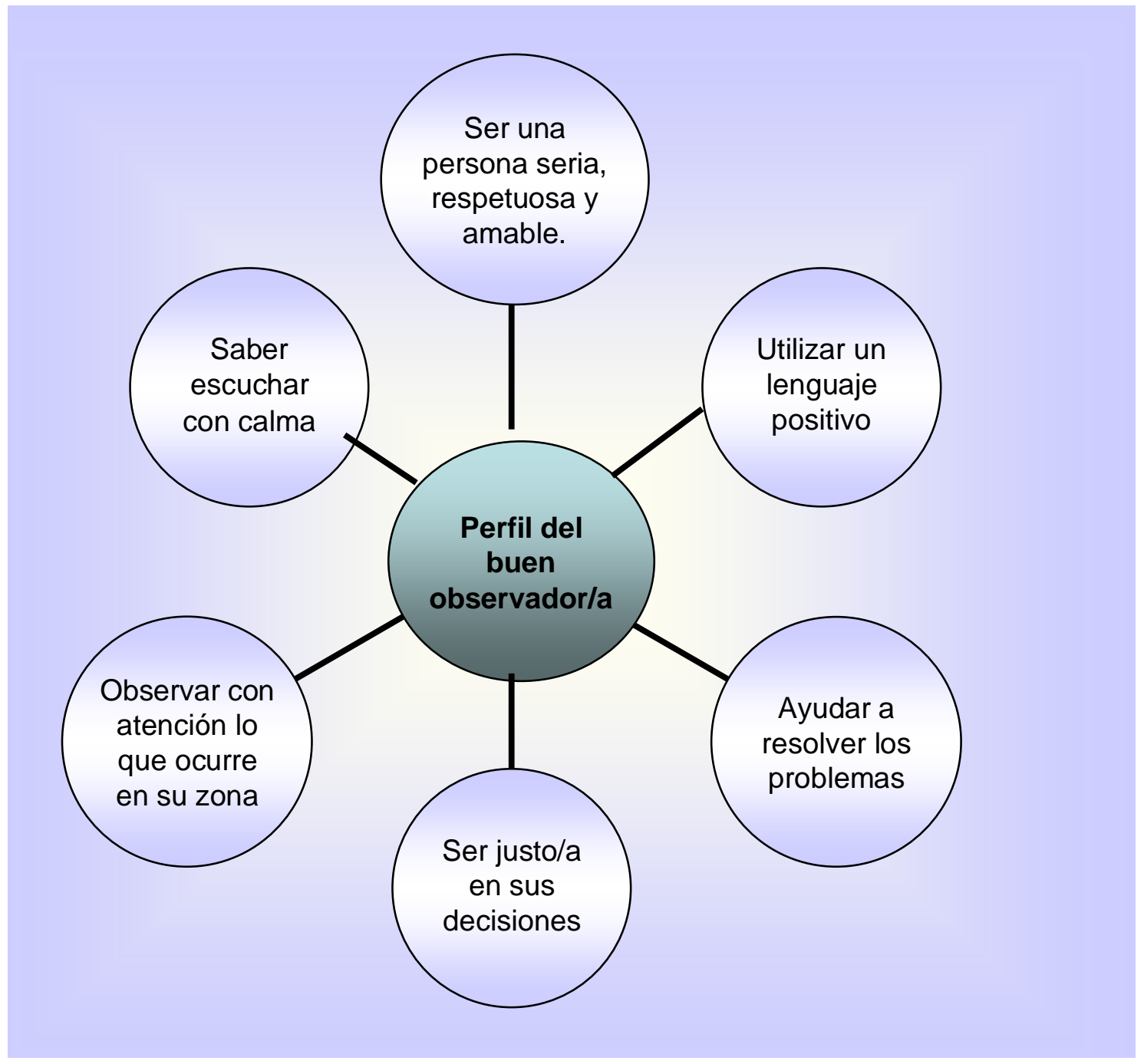

Figura 3.- Perfil del buen observador/a. 
del Profesorado de Lanzarote y por el Gobierno de Canarias a través de la teleformación), así como charlas, talleres y otras actividades relacionadas. Esta formación es permanente y continua, por lo que ha día de hoy seguimos formándonos en este ámbito.

Segundo paso: Presentación a la comunidad educativa y propuestas de mejora.

- Se llevan a cabo diferentes reuniones con el equipo directivo, el claustro y el consejo escolar a fin de presentar a segunda fase del proyecto y concretar las acciones a realizar.

- Se procede a reunir a la Junta de Delegados/as para que sirva de nexo de unión entre la coordinación del proyecto y el alumnado. Se les informa de las acciones que se van a llevar a cabo y se anotan las valoraciones y propuestas de mejora que ofrecen al respecto.
- Se convocan asambleas por tutorías, en las que mediante el diálogo se sondea al alumnado sobre aquellas cosas que creen que deben ser observadas durante el recreo a fin de que este transcurra sin ningún tipo de incidente, el perfil que piensan que deben reunir los observadores/as y se piden voluntarios/as para desempeñar el rol de observador/a entre los alumnos de cuarto y quinto curso de Primaria (figura 3).

Tercer paso: Selección y formación del alumnado.

- Acto seguido el Equipo Directivo y el Coordinador, con la colaboración de los tutores/as de dichos cursos, seleccionan a aquellos alumnos/as que más se ajustan al perfil y que mejor pueden desempeñar a priori dicha función.

- Una vez formados los grupos, se aseso-

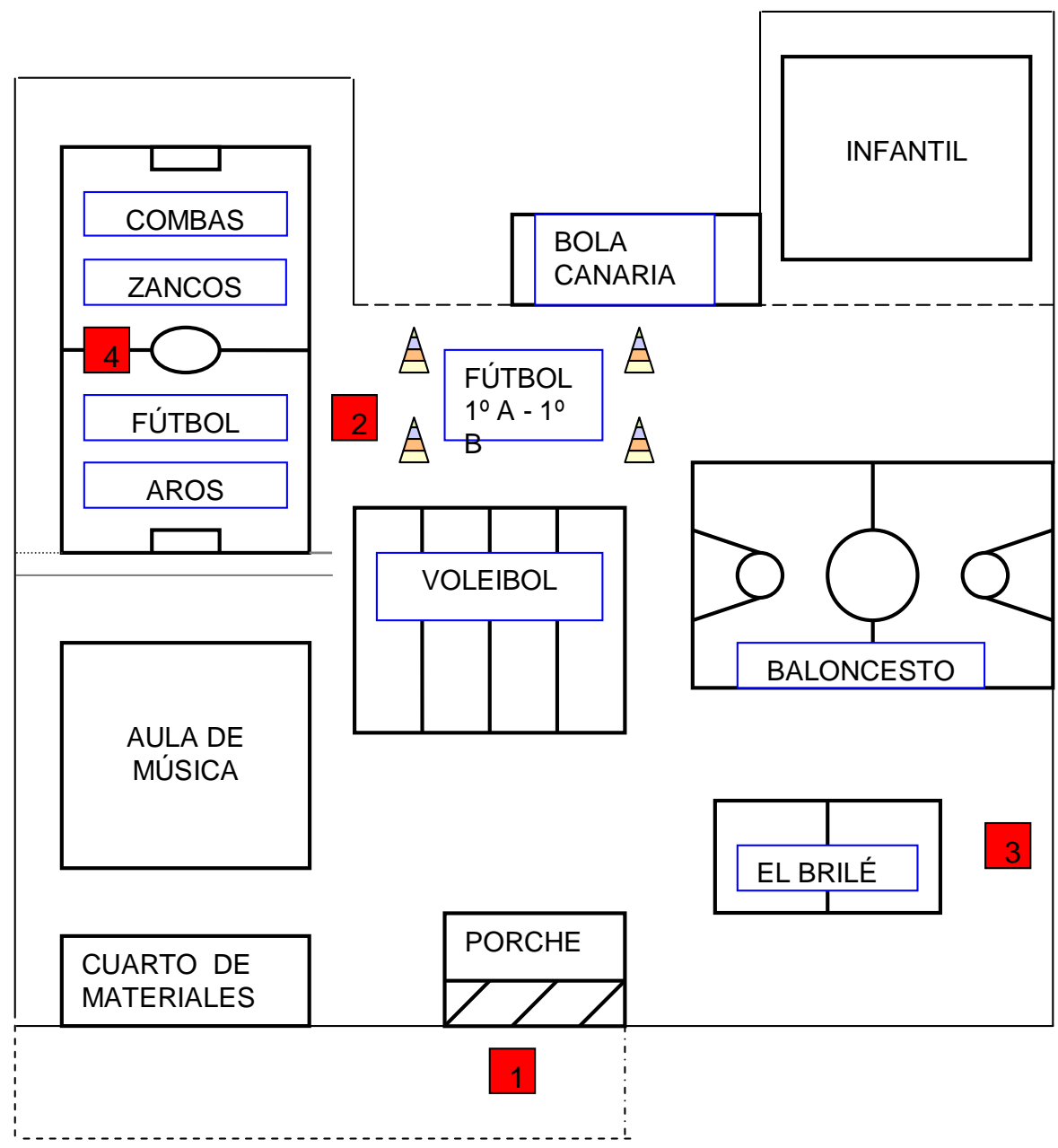

Figura 4.- Distribución de las zonas del patio para la observación. 
ran, con una formación dirigida principalmente al desarrollo de habilidades sociales básicas para la escucha activa y la resolución de conflictos leves, pues su principal función es la de observar y registrar lo que acontezca en el patio.

\section{Cuarto paso: Distribución de zonas.}

- El coordinador del proyecto elabora las zonas del patio a observar, que son cuatro claramente diferenciadas, donde se colocará a cada observador/a, teniendo unas pautas específicas para esa zona asignada. Para ello se elaboran cuatro libretas de registro de diferentes colores que corresponden con cada una de las zonas en las que se deben registrar las incidencias que ocurran (figura 4).

\section{Quinto paso: Plan de acción.}

- Antes de la puesta en marcha, última reunión con los observadores/as para concretar todos los aspectos, resolución de dudas y cuestiones varias.

- Para dar una mayor difusión y facilitar el acceso a la información de todos/as, se crea un espacio en un lugar visible del colegio donde se cuelgan diferentes carteles informativos con las pautas de convivencia, las zonas de los observadores, los horarios de los grupos de los observadores/as y el nombre de los alumnos/as participantes, etc.

- Para que el alumnado que juega en el patio pueda localizar rápidamente donde se encuentran los observadores/as, se han adquirido unas camisetas de color rojo en las que se le ha serigrafiado el término observador/a en la espalda. Esto ayuda tanto a la rápida localización como a la propia valoración de la labor que realizan estos alumnos/as en el centro (figura 5).

\section{Sexto paso: Seguimiento y evaluación.}

- Todas las semanas se lleva a cabo un vaciado de los registros obtenidos y, a final de cada mes, se valoran los resultados y se toman las medidas oportunas.

- Puesto que esta fase se ha comenzado a desarrollar de forma práctica el 18 de enero, aún es pronto para ver los resultados, pues ahora se están puliendo los errores de planificación y ejecución, así

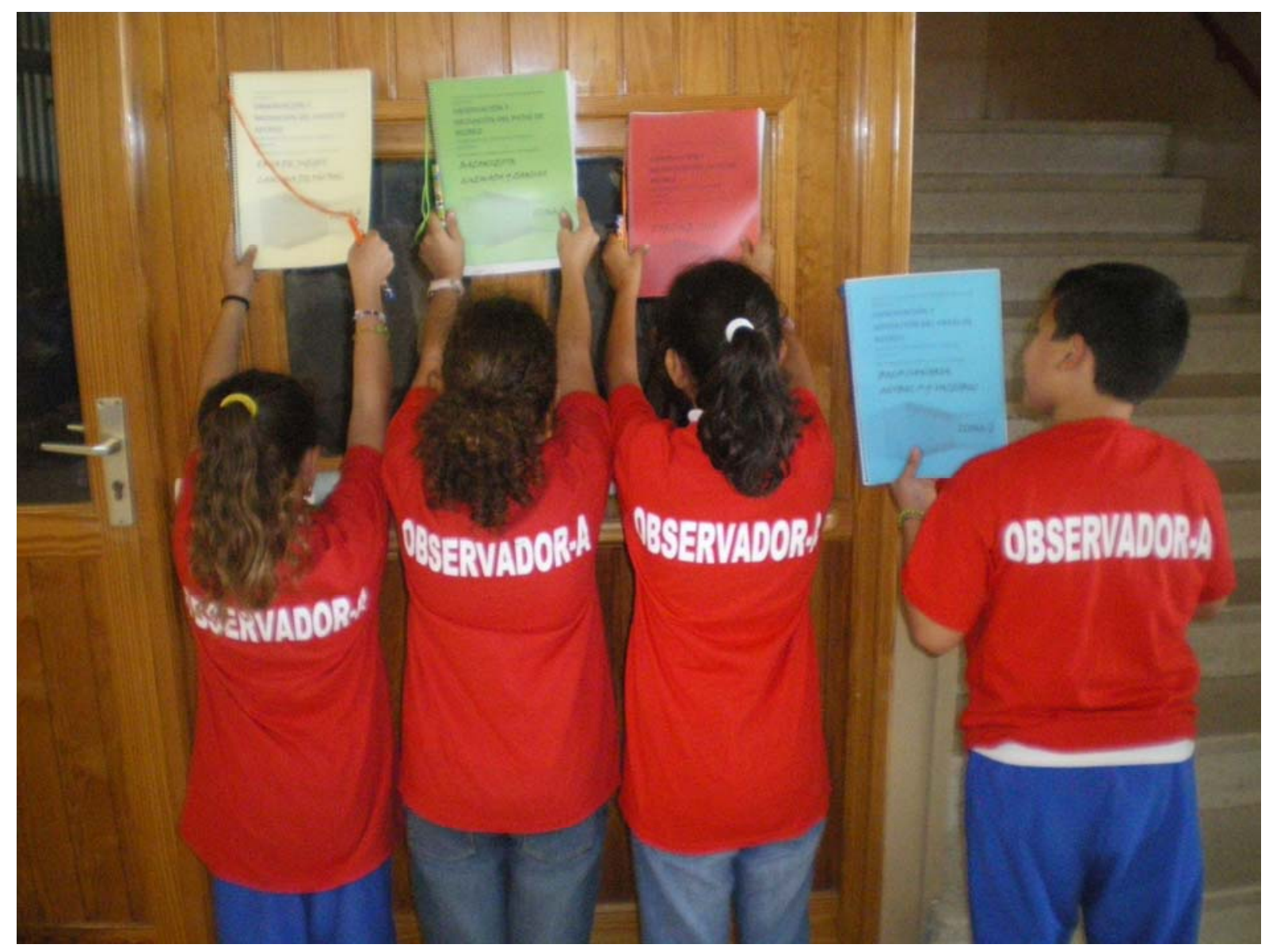

Figura 5. - Varios de los alumnos y alumnas seleccionados/as como observadores/as. 
como dando cabida a las propuestas de mejora que van surgiendo. No obstante, las primeras valoraciones son muy positivas, ya que el alumnado está participando con agrado en toda la experiencia.

- Al igual que la fase anterior, pretendemos que esta fase no sea cerrada, dejando así siempre el camino abierto a posibles mejoras, por lo que será necesario llevar a cabo un seguimiento continuo.

\section{4.- REPERCUSIÓN SOBRE LA COMUNIDAD EDUCATIVA.}

Entendemos por comunidad educativa a todos aquellos estamentos relacionados directa o indirectamente con la educación. Por ello consideramos como elementos primarios al alumnado, al profesorado y las familias, pero la repercusión de la educación incide en toda la sociedad en general.

$\square$ En el alumnado este proyecto repercute de dos formas claramente diferenciadas:

- Para el alumnado seleccionado como observador conlleva una mejora y aumento de la autoestima, la responsabilidad, la cooperación, el trabajo en equipo y el diálogo, aprendiendo a rechazar todo tipo de discriminación y agresión verbal o física.

- Para el alumnado receptor del proyecto (incluidos los observadores, ya que al existir diferentes grupos no todos los días desarrollan dicha función): en la medida que aumenta la diversión en el patio ante la variedad de actividades lúdicas jugadas, disminuye la generación de conflictos, aumenta la adquisición y afianzamiento de las normas conductuales (trasladables fuera del colegio y aplicables a la vida diaria) y se mejora el clima de convivencia (minimizándose los posibles casos de acoso escolar y/o violencia dentro y fuera del recinto escolar).

\section{En el profesorado:}

- Se minimiza la implicación del profesorado en la resolución de conflictos (ya que el desarrollo del proyecto conlleva una reducción importante de los mismos) y las relaciones maestro-alumno mejoran tanto en el patio como en las aulas (por lo que se puede trabajar más y mejor). Además los cursos y talleres realizados por el profesorado sobre convivencia en- riquecen su formación, pudiendo así garantizar una mayor calidad en la enseñanza.

\section{En las familias:}

- La mejora de la convivencia en el centro y la adquisición de normas de conducta incide directamente en el comportamiento del alumno/a en casa, lo que facilita una relación más cordial entre todos los miembros de la familia. Así mismo este proyecto ofrece tranquilidad y confianza en padres, madres y tutores/as del alumnado al observar la implicación del profesorado en la mejora de la educación de sus hijos, lo que repercute de forma muy positiva en la relación familia-profesor.

$\square$ En la sociedad:

- Si formamos alumnos/as responsables y respetuosos ofrecemos a la sociedad personas educadas y comprometidas, capaces de ejercer debidamente la ciudadanía, tal como se pretende, entre otros aspectos, con la adquisición de las competencias básicas; según el Decreto 126/2007, de 24 de mayo, por el que se establece la ordenación y el currículo de la Educación Primaria en la Comunidad Autónoma de Canarias, "Se entiende por competencias básicas el conjunto de conocimientos, habilidades y actitudes que debe alcanzar el alumnado al finalizar la enseñanza básica para lograr su realización y desarrollo personal, ejercer debidamente la ciudadanía, incorporarse a la vida adulta de forma plena y ser capaz de continuar aprendiendo a lo largo de la vida" (BOC ${ }^{\circ}$ 112, 2007, 12664).

Las competencias básicas del sistema educativo español surgen en base a la "Recomendación del Parlamento Europeo y del Consejo de 18 de diciembre de 2006 sobre las competencias clave para el aprendizaje" (2006/962/CE), pero han sido adaptadas a nuestra realidad educativa, quedando establecidas las siguientes: competencia en comunicación lingüística, competencia matemática, competencia en el conocimiento y en la interacción con el mundo físico, tratamiento de la información y competencia digital, competencia social y ciudadana, competencia cultural y artística, competencia para aprender a aprender y autonomía e iniciativa personal. No obstante, algunas comunidades autónomas han introducido 
cambios en la denominación de algunas de ellas, no en la esencia de las mismas, como por ejemplo Andalucía, en la cual según el Decreto 230/2007, de 31 de julio, por el que se establece la ordenación y las enseñanzas correspondientes a la educación primaria en Andalucía, se establecen: competencia en comunicación lingüística, competencia en razonamiento matemático, competencia en el conocimiento e interacción con el mundo físico y natural, competencia digital y tratamiento de la información, competencia social y ciudadana, competencia cultural y artística, competencia y actitudes para seguir aprendiendo de forma autónoma a lo largo de la vida y competencia para la autonomía e iniciativa personal (BOJA ${ }^{0}$ 156, 2007, 10-11).

\section{5.- CONCLUSIONES.}

La optimización del patio y el préstamo de materiales específicos del área de Educación Física en el tiempo del recreo nos ofrecen la posibilidad realizar mayor cantidad de actividades físico-deportivas, de educar al alumnado en valores y de favorecer las relaciones interpersonales entre iguales, contribuyendo así fundamentalmente con la competencia en comunicación lingüística, la competencia en el conocimiento e interacción con el mundo físico y natural, y con la competencia social y ciudadana. En palabras de Schulman (2002), "las escuelas pueden contribuir a la mejora de la relaciones interpersonales fomentando entre sus alumnos valores morales basados en principios de igualdad, preocupación, tolerancia y empatía por los sentimientos de los demás”. No obstante, para lograr óptimos resultados, será imprescindible contar con el apoyo y el consenso del resto del profesorado e implicar de forma activa al alumnado, convirtiéndose así estos últimos en los protagonistas de su propio aprendizaje.

\section{6.- REFERENCIAS BIBLIOGRÁFICAS.}

Baena, A., Calvo, J.F. \& Martínez, M. (2009). Una experiencia didáctica en metodología integrada: cinco estilos de enseñanza a través de la bicicleta de montaña, patines y monopatines en E.S.O. Espiral. Cuadernos del profesorado 2(3), 41-48.

Cowie, H. \& Fernández F.J. (2006). Ayuda entre iguales en las escuelas: desarrollo y retos. Revista Electrónica de Investigación Psicoeducativa, 4(2), 291-310.

Decreto 126/2007, de 24 de mayo, por el que se establece la ordenación y el currículo de la
Educación Primaria en la Comunidad Autónoma de Canarias (BOC, 112 de 6 de junio).

Decreto 230/2007, de 31 de julio, por el que se establece la ordenación y las enseñanzas correspondientes a la Educación Primaria en Andalucía.

Escamilla, A., Lagares, A.R., \& Fraile, J.A. (2006). La LOE: perspectiva pedagógica e histórica: Glosario de términos esenciales. Barcelona: Graó.

Ley 9/1999, de 18 de noviembre, de Solidaridad en la Educación (BOJA, 140 de 2 deDiciembre).

Ley Orgánica 2/2006, de 3 de mayo, de Educación (BOE, 106 de 4 de mayo).

Recomendación del Parlamento Europeo y del Consejo de 18 de diciembre de 2006 sobre las competencias clave para el aprendizaje (2006/962/CE)

Schulman, M. (2002). How we become moral. The Sources of Moral Motivation. En, C.R. Snyder \& S.L. López (Eds.), Handbook of Positive Psychology (pp. 498-512). Oxford: Oxford University Press.

Sebastiani i Obrador, E.M. \& Delgado Noguera, M.A. (2009). Identificación y formación de las competencias del profesorado de Educación Física. En D. Blázquez \& E.M. Sebastiani (Eds.) Enseñar por competencias en Educación Física (pp. 191-208). Barcelona: Inde.

Vila, E. (2009). Educar para la tolerancia, educar para la convivencia. Espiral. Cuadernos del profesorado, 2(4), 43-47.

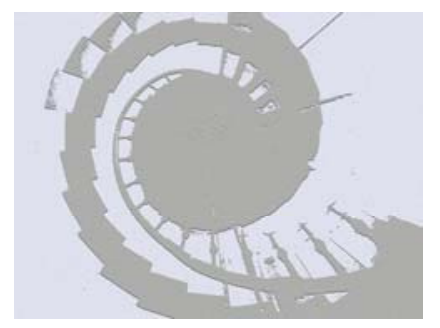

